\title{
Diagnostic criteria and genetics of the PEHO syndrome
}

\author{
Mirja Somer
}

\begin{abstract}
The PEHO syndrome (progressive encephalopathy with oedema, hypsarrhythmia, and optic atrophy) is a recently recognised disorder of unknown biochemical background. Diagnostic features have been found in neuroradiological and neuropathological studies, which show characteristic severe cerebellar atrophy. In combined neuroradiological and ophthalmological studies, 10 out of 21 possible PEHO patients fulfilled the criteria for true PEHO syndrome. All were abnormal at birth showing hypotonia, drowsiness, or poor feeeding. Head circumference was normal, but usually dropped to 2 SD below average during the first year of life. Visual fixation was either absent from birth or lost during the first months of life. Nine patients had peripheral oedema in early childhood. The mean age of onset of infantile spasms was 4.9 months. All patients were extremely hypotonic and no motor milestones were reached. Patellar reflexes were brisk. Brain stem and somatosensory evoked potentials were abnormal in each case studied, cortical responses of somatosensory evoked potentials could not be elicited, and motor conduction velocities became delayed with age.
\end{abstract}

Altogether 19 PEHO patients were found in 14 Finnish families. Autosomal recessive inheritance is likely.

( 7 Med Genet 1993;30:932-6)

The PEHO syndrome (progressive encephalopathy with oedema, hypsarrhythmia, and optic atrophy) was recently described by Salonen et $a l^{1}$ in 14 patients from 11 Finnish families. There was no chromosomal abnormality or biochemical marker for the disorder, and initially the diagnosis was based on typical medical history and dysmorphic features. Subsequently, characteristic neuropathological features were found in eight PEHO patients. ${ }^{2}$ There was an extremely atrophic cerebellum, which showed severe neuronal loss from the granular layer, deformed and misaligned Purkinje cells, and a narrow molecular layer. Accordingly, cerebellar atrophy in neuroimaging studies became the most important clinical criterion for the PEHO syndrome, ${ }^{3}$ together with optic atrophy confirmed by visual evoked potentials. ${ }^{4}$ This article describes additional clinical features of the PEHO syndrome and proposes the clinical diagnostic criteria. In addition, genetic studies of all PEHO syndrome families in Finland are reported.

\section{Patients and methods}

Seven patients from the original study by Salonen $e t a l^{1}$ were available for further study. Fourteen additional patients with suspected PEHO syndrome were found through clinical geneticists and neuropaediatricians and by personally visiting the 15 Finnish central institutions for the mentally retarded. The inclusion criteria are shown in table 1.

To differentiate the patients with true PEHO syndrome, all patients were evaluated by brain CT scan or magnetic resonance imaging, and they had neuro-ophthalmological examination complemented by electroretinogram and flash visual evoked potentials.

Brain stem auditory evoked potentials were obtained by stimulating each ear with 100 to $110 \mathrm{~dB}$ intensity sound pressure level. The recording was obtained between the vertex and the mastoids. The analysis time was $10 \mathrm{~ms}$ after the stimulus. Somatosensory evoked potentials were obtained by stimulating the median nerve at the wrist. The stimulation intensity was just above the motor threshold. Motor nerve conduction velocities were measured with standard techniques.

Routine laboratory investigations were performed, including blood lactate and pyruvate values, plasma level of postprandial ammonium, serum transaminases, creatine kinase, copper, and caeruloplasmin. Peripheral blood smears were examined for vacuolated lymphocytes. Urinary screening studies for storage disorders of glycoproteins, glycolipids, and glycosaminoglycans were performed. Protein, glucose, lactate, and pyruvate levels were measured in cerebrospinal fluid. Special metabolic investigations included the determination of plasma and urine levels of amino acids by ion exchange chromatography, urinary organic acids by gas chromatography, and determination of urinary arylsulphatase and orotic acid. Urinary oligosaccharides were studied by thin layer chromatography. Serum long chain fatty acids and phytanic acid were measured in five patients and serum biotini-

\section{Table 1 Inclusion criteria of the patients.}

A Features present in all patients
Seizure onset at $2-52$ weeks of life*

Seizure onset at 2-52 weeks of life
Infantile spasms/hypsarrhythmia

Infantile spasms/hypsarrhythmia

Profound hypotonia with no head support or ability to sit unsupported

Poor/absent visual fixation from the first months of life

B Additional features, present in most patients Subcutaneous peripheral and facial oedema

Microcephaly developing by 12 months of age

Dysmorphic features (epicanthic folds, midfacial hypoplasia, protruding ear lobes, receding chin, tapering fingers)

Pale optic discs

* One patient had generalised seizures from birth
Helsinki, Finland.

Received 18 February 1993. Revised version accepted 23 May 1993. 
dase in one patient. Carbohydrate deficient glycoprotein determination 5 was done on two patients.

The genetic study included all the neuropathologically ${ }^{2}$ and clinically diagnosed PEHO patients and their similarly affected sibs. Family histories were obtained from the parents and hospital records. If there was a relative with mental retardation, further information about the aetiology was acquired from the hospital records. The birth places of the grandparents and the total number of children in the families in October 1992 were confirmed through the population register. Information on ancestors covering four to six generations was obtained if a maternal and a paternal grandparent or grandparents of two different families were born in the same or the neighbouring community. A segregation analysis was performed with corrections for truncate complete ascertainment by Apert's a priori method, and for truncate single ascertainment by omitting one affected child from each sibship. ${ }^{6}$

\section{Results}

Serial neuroimaging studies in 12 of 21 patients showed early progressive, predominantly cerebellar and brain stem atrophy, while nine patients had mostly non-progressive, predominantly supratentorial abnormalities. $^{3}$ Two of the 12 patients were excluded from the study because they had no optic atrophy. The detailed results of the neuroradiological and ophthalmological studies will be reported separately. ${ }^{34}$ The remaining 10 patients were considered to have the PEHO syndrome.

\section{CLINICAL COURSE}

Eight of the 10 patients were born after a normal pregnancy. Two pregnancies were complicated near term by mild maternal hypertonia and oedema. No polyhydramnios was observed and fetal movements were not reported to be weak by any of the mothers. Three infants were in breech presentation. Two of them and two other patients were delivered by caesarean section. All infants were born at term except one, who was born at 37 weeks of gestation. At birth, two newborns responded poorly and had respiratory problems necessitating short oxygen administration. The other eight newborns had Apgar scores ranging from 8 to 10 .

Hypotonia and other abnormal features were observed in all infants during the first days of life (table 2). One newborn had oedema in the lower abdomen, prepuce, and lower limbs, and another in the dorsum of the feet. None of the newborns had seizures during the first two weeks of life. Definite hypotonia was noted in all patients before the onset of infantile spasms. Visual fixation was always lost by the time the seizures began. Pupillary reactions to light became sluggish and there was typical deviation of gaze sideways and upwards. Infantile spasms were noted at 2 to 5
Table 2 Clinical history of the 10 PEHO patients.

\begin{tabular}{lc}
\hline Abnormal at birth & 10 \\
Findings at birth & \\
Hypotonia & 7 \\
Poor feeding & 6 \\
Abnormal cry & 5 \\
Oedema & 2 \\
Drowsiness & 2 \\
Hypothermia & 2 \\
Dysmorphic facies & 3 \\
Oedema in early childhood & 9 \\
Onset of infantile spasms & \\
$\quad<6$ months & 8 \\
6-11 months & 2 \\
Loss of visual fixation & \\
From birth & 5 \\
From 2-9 months of age & 5 \\
Absent psychomotor development & 10 \\
\hline
\end{tabular}

months of age in eight patients, and at 9 and 10 months in two patients, the mean age of onset being 4.9 months. After the infantile spasms all the patients continued to have seizures, often compatible with the Lennox-Gastaut syndrome.

No motor milestones were reached. The patients could not roll over or sit unsupported, and they showed severe head lag at all ages. The patellar and ankle stretch reflexes were exaggerated by 24 months of age in all patients except one. There was often ankle clonus and a positive Babinski sign. Athetoid movements of the limbs were seen in three patients and tongue thrusting in four. The patients had very little voluntary movement in the extremities. Increasing spasticity with rigidity of the joints was noted from 2 to 4 years of age, with contractures mainly in the knees and ankles. The patients could not chew their food, but they swallowed normally. They had constipation and decreased frequency of urination.

The patients spoke no words at any age. However, they communicated by various vocal sounds, and responded with a smile when they were spoken to. Oedema, which was not associated with adrenocorticotrophin (ACTH) treatment, was observed in the facies and on the dorsal side of the hands and feet (fig 1A), often extending to the elbow and knee. After early childhood the oedema was noted to disappear, first from the facies and later from the limbs.

\section{PHYSICAL FINDINGS}

\section{Growth}

The mean birth weight, length, and head circumference was average, ${ }^{7}$ the weights ranging from 2800 to $3970 \mathrm{~g}$, lengths from 49 to $52 \mathrm{~cm}$, and head circumferences from 35 to $37 \mathrm{~cm}$ at term. In one patient, however, all these measurements were 3 SD below the mean. The heights fell to 2 SD below normal by the second year of life, and thereafter gradually to 3 to $5 \mathrm{SD}$ below normal, ${ }^{7}$ and the patients also became very slim. During infancy, the mean head circumference dropped to $-2 \mathrm{SD}$, remaining on the curve thereafter; thus the head was small in relation to age but not in relation to body size. 


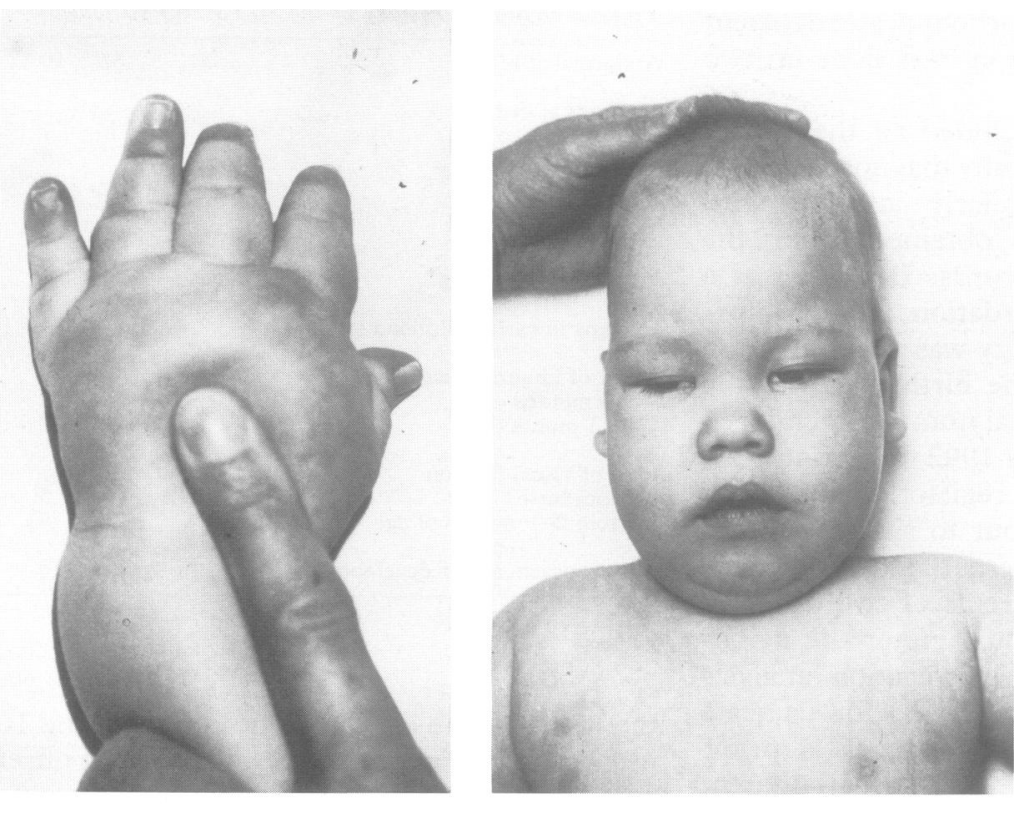

(A)

Figure $1 A$ boy with the PEHO syndrome at 9 months of age. The hands show oedema, and the nails are deep set and distorted $(A)$. Note the narrow forehead, epicanthic folds, outward turning ear lobes, and small chin (B).
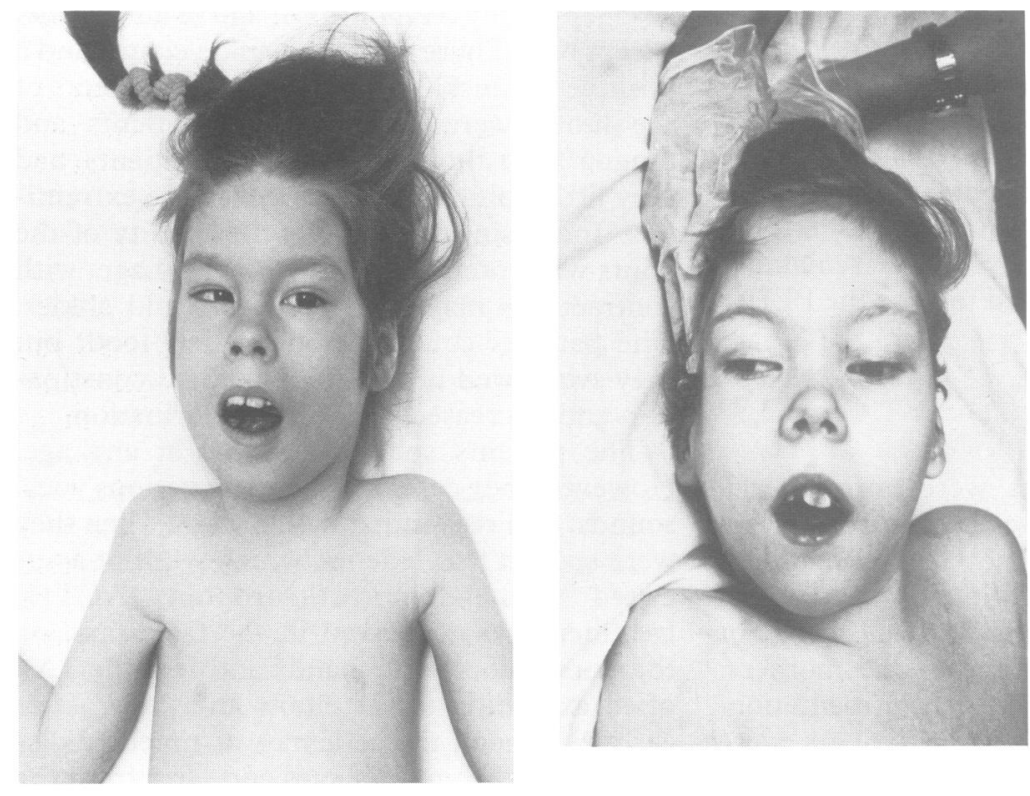

(A)

Figure 2 The 8 year old girl ( $A$ ) has the PEHO syndrome. The 12 year old girl B) shows very similar facial features, but the PEHO syndrome was ruled out by neuroradiological and, subsequently, by neuropathological examinations.

\section{Dysmorphic features}

Epicanthic folds, short nose with anteverted nostrils, outward turning ear lobes, and small chin were already present in some patients in the newborn period. The features were most typical during the first years of life (fig 1B). Characteristic deviation of the eyes and constantly open mouth contributed to the typical facial expression (fig 2A). The palate was high. Although none of the patients received phenytoin, the gingivae were hypertrophied (fig 3). The fingers were tapering, the limbs were slender, and articular contractures appeared after 2 to 3 years of age.
NEUROPHYSIOLOGICAL FINDINGS

Brain stem auditory evoked potentials were abnormal in each of the nine patients studied. One patient showed absence of all waves, which was consistent with the hearing deficiency in this patient. In the other patients the latency to wave 1 was normal and to waves 3 and 5 delayed.

Somatosensory evoked potentials were recorded in four patients at $0 \cdot 8,2 \cdot 7,8 \cdot 2$, and 12.7 years of age. The cortical responses were absent in each of them, while the peripheral responses were preserved.

Motor conduction velocities were normal in two of the youngest patients, but slower than normal in all patients that were 2 years of age or older, and there was a progressive decrease of the conduction velocities with age (table 3 ).

\section{SKELETAL AND VISCERAL FINDINGS}

The skull was narrow and flattened at the sides. The spine showed thoracic or thoracolumbar scoliosis, sometimes combined with kyphosis. The long bones showed poor modelling, extreme slenderness, and osteopenia in all patients except the youngest. The head of the femur was subluxated in five patients. Some showed signs of previous fractures in tubular bones. The heart was of normal size in all the patients, and abdominal ultrasound showed the liver and spleen to be of normal size in all the patients. The kidneys appeared normal; in particular, there were no cysts.

\section{LABORATORY FINDINGS}

No significant haematological or biochemical abnormalities were detected. No patient showed signs of hepatic or renal disease and the electrolyte values were normal. There was no evidence of any mitochondrial, peroxisomal, or lysosomal disorder in any of the patients.

\section{PEHO-like patients}

Eleven out of 21 patients were excluded from the study by neuroradiological and ophthalmological studies. At birth, one infant had generalised convulsions with a suppressionburst pattern on EEG, and two infants were microcephalic. Six other patients developed disproportionate microcephaly in early childhood. Although all the infants were described as floppy, hypotonia was overshadowed by hypertonia and opisthotonus in five patients. Oedema was noted in eight patients; in four, however, it was temporary and coincided with ACTH treatment. Two other patients showed oedema in late childhood but not in infancy. Pupillary reactions to light could be elicited in seven patients. Seven patients had normal or weak reflexes. On the basis of facial features it was not possible to distinguish a PEHO syndrome patient from one with a PEHO-like disorder (fig 2B).

Brain stem evoked potentials indicated normal brain stem function in six of eight patients 


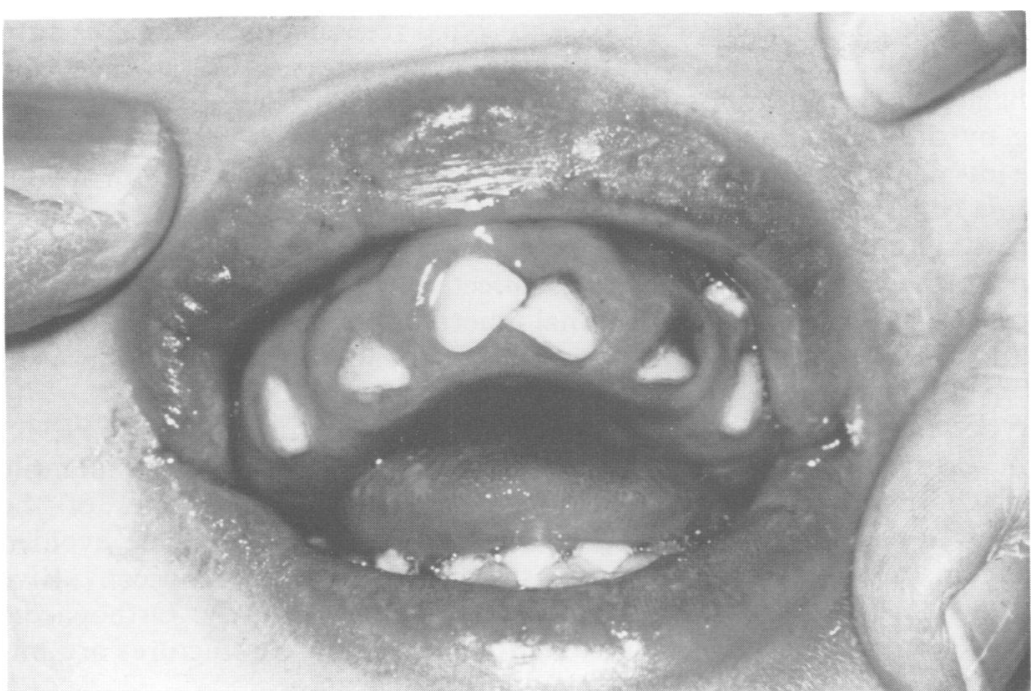

Figure 3 Severe gingival hypertrophy in a 4 year old PEHO patient.

Table 3 Motor nerve conduction velocities in nine PEHO patients.

\begin{tabular}{lcc}
\hline Age $(\mathrm{y})$ & \multicolumn{2}{c}{ Motor nerve conduction velocity $(\mathrm{ms})$} \\
\cline { 2 - 3 } & Ulnar nerve & Peroneal nerve \\
\hline $0 \cdot 8$ & $34(>38)^{*}$ & $37(>32)$ \\
1.9 & $48(>48)$ & $54(>38)$ \\
$2 \cdot 8$ & $36(>50)$ & $38(>42)$ \\
$3 \cdot 3$ & $41(>52)$ & $37(>44)$ \\
$3 \cdot 9$ & $41(>52)$ & $42(>44)$ \\
$7 \cdot 6$ & $36(>52)$ & $36(>44)$ \\
$8 \cdot 1$ & $38(>52)$ & $38(>44)$ \\
$8 \cdot 2$ & $28(>52)$ & $29(>44)$ \\
$12 \cdot 7$ & $36(>52)$ & $26(>44)$ \\
\hline
\end{tabular}

${ }^{*}$ Normal for age in parentheses.

studied. Cortical responses of somatosensory evoked potentials were elicited in four of five patients studied. Motor nerve conduction velocities were normal in nine patients and slightly delayed in one.

\section{Genetics}

The 14 PEHO syndrome families had a total of 32 children, of whom six boys and 13 girls were affected (fig 4). The sex ratio (male to female) of the affected children was 0.46 . The disorder was of equal severity in males and females, and there were no affected persons in older generations. The patients had eight half sibs altogether, none of whom was affected with the PEHO syndrome. The mothers of
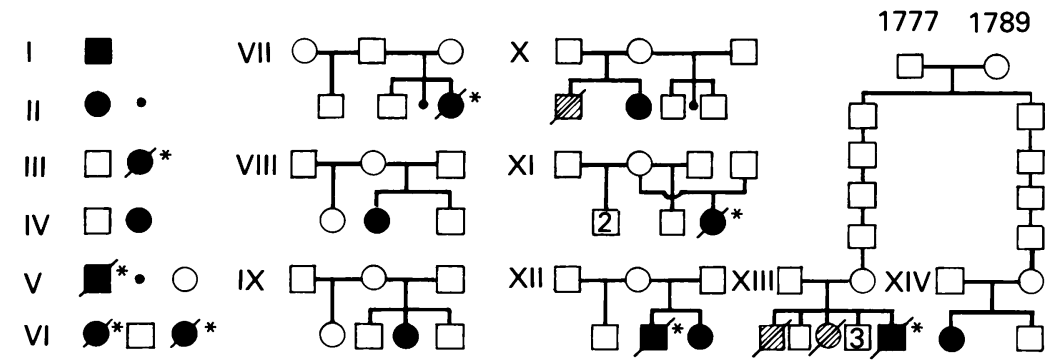

Figure 4 The 14 sibships with 19 PEHO patients. Families XIII and XIV were related. Solid symbols mark the patients diagnosed as having the PEHO syndrome. Neuropathologically studied cases are marked by an asterisk. Hatched symbols mark the dead, similarly affected sibs, who were not examined neuropathologically.
Table 4 Clinical criteria for the PEHO syndrome.

Necessary criteria

(1) Infantile, usually neonatal hypotonia

(2) Convulsive disorder manifesting with myoclonic jerking and infantile spasms

(3) Profound psychomotor retardation with severe hypotonia; absence of motor milestones and speech

(4) Absence or early loss of visual fixation with atrophy of optic discs by 2 years of age; normal electroretinogram extinguished visual evoked potentials

(5) Progressive brain atrophy in neuroimaging studies, particularly in the cerebellum and brain stem; milder supratentorial atrophy

Supportive criteria

(1) Subtle dysmorphic features with narrow forehead, epicanthic folds, short nose, open mouth, receding chin, and tapering fingers

(2) Oedema of the face and limbs, especially in early childhood

3) Brisk tendon reflexes in early childhood

4) Abnormal brain stem auditory evoked potentials

5) Absent cortical responses of somatosensory evoked potentials

Slow nerve conduction velocities in late childhood

(7) Dysmyelination in magnetic resonance imaging

Features that argue against the PEHO syndrome

(1) Microcephaly at birth

(2) Abnormal gyral formation in neuroradiological studies

(2) Abnormal gyral formation in neuror

(4) Reappearance of visual contact after cessation of

Reappearance of
infantile spasms

(5) Hepato/splenomegaly or storage disorder in histological studies

two families (fig 4, families XIII and XIV) were fourth cousins. No parental consanguinity was found in any of the families; however, one maternal and one paternal grandparent of family XIII were born in the same community, and in family VI all four grandparents came from the same sparsely inhabited rural community. The birth places of the grandparents showed some accumulation in southeastern, central, and western Finland. No definite regional clustering, however, was noted. In the 14 sibships, the proportion of affected sibs was 0.290 (SE 0.039) when corrected for truncate complete ascertainment, and 0.278 (SE 0.011) when corrected for truncate single ascertainment.

Although this study does not show the true incidence of PEHO syndrome in Finland, a minimum incidence can be estimated. The incidence calculated for the 15 year period 1974 to 1988 was $1: 74000$, which is a little less than one new case every year.

\section{Discussion}

When the PEHO syndrome is suspected, neuroimaging studies are crucial in confirming the diagnosis, together with careful ophthalmological evaluation. Eleven out of 21 patients in this study had quite similar clinical histories and physical findings, but their neuroradiological or neuro-ophthalmological findings differentiated them from the true PEHO syndrome. Infantile brain atrophy, which originates in the cerebellum and progresses predominantly in the infratentorial regions, is the major diagnostic criterion for the true PEHO syndrome, ${ }^{3}$ together with optic atrophy. ${ }^{4} \mathrm{Ab}-$ normal brain stem and somatosensory evoked potentials as well as progressive slowing of nerve conduction velocities support the diagnosis (table 4).

No metabolic defect was found in laboratory studies; therefore neither prenatal nor carrier 
diagnosis is available. Molecular genetic studies will probably be needed to solve this problem and to confirm the diagnostic classification of the patients. Judging by the four affected sibships in this study the intrafamilial variability of the PEHO syndrome is narrow.

Although it is often the dysmorphic features that raise the suspicion of the PEHO syndrome, they proved to be non-specific (fig 2). Many of the facial features were associated with developing microcephaly, extreme floppiness, and oedema. Hypotonia was usually observed from birth, whereas hypertonia was not noted in any of the newborns.

Nine of ten patients showed oedema in infancy, the cause of which remained unknown. Infantile subcutaneous oedema has been observed in some lysosomal storage disorders, such as galactosialidosis and infantile $G_{M 1}$ gangliosidosis, ${ }^{8}$ but histopathological studies in the PEHO syndrome patients showed no evidence of any storage disease. ${ }^{2}$ Distorted nails (fig 1A) suggest fetal lymphoedema, ${ }^{9}$ but redundant skin in the neck was not seen in any of the patients.

No disorder identical to the PEHO syndrome has been reported elsewhere. It appears to be one of the rare autosomal recessive disorders that are overrepresented in Finland, because of the national and regional isolation of an originally small population. ${ }^{10}$ In this study, patients with possible PEHO syndrome were collected nationwide from many different sources, and 14 families with 19 affected children were finally identified. The grandparental birth places of the families, however, did not show clustering into a few regions, which is the case in several other hereditary disorders typical of the Finns. ${ }^{11}$ This suggests that the gene is not very rare in the population and that more patients may exist in Finland. In addition, there are now patients with possible PEHO syndrome in several other countries, including Great Britain (Chitty et al, paper presented at the 5th Manchester Birth Defects Conference, 1992).

The proportion of affected sibs was 0.290 (SE 0.037) and 0.278 (SE 0.011), when corrected for truncate complete and single ascertainment, respectively. ${ }^{6}$ Both are above the expected 0.25 ratio, although $1 \mathrm{SE}$ for truncate complete ascertainment $(0 \cdot 253)$ is close to that ratio. Autosomal recessive inheritance is supported by several factors. Affected male and female sibs in three families showed no difference in terms of severity of the disorder. The mothers of two families were fourth cousins. None of the eight maternal half sibs of PEHO syndrome patients in families VIII to XII was affected (fig 4), which argues against maternal inheritance. The unequal sex ratio of the patients is probably the result of the small series.

There is no specific treatment for the PEHO syndrome. ACTH medication for infantile spasms was not successful in any of the patients, and it should probably be avoided because of the side effects. Anticonvulsive therapy is needed permanently. Orthopaedic measures for preventing contractures are important.

I thank all those clinicians who referred patients to this study. I am grateful to Professor Reijo Norio for his comments on the manuscript. This study was supported by the Rinnekoti Research Foundation, Arvo and Lea Ylppö Foundation, and Päivikki and Sakari Sohlberg Foundation, Finland.

1 Salonen R, Somer M, Haltia M, et al. Progressive encephalopathy with edema, hypsarrhythmia, and optic atrophy (PEHO syndrome). Clin Genet 1991;39:287-93.

2 Haltia $M$, Somer $M$. Infantile cerebello-optic atrophy: neuropathology of the progressive encephalopathy syndrome with hypsarrhythmia and optic atrophy (the PEHO syndrome). Acta Neuropathol 1993;85:241-7.

3 Somer M, Salonen O, Pihko H, Norio R. PEHO syndrom (progressive encephalopathy with edema, hyps-
arrhythmia, and optic atrophy): neuroradiologic findings. afNR 1993;14:861-7.

4 Somer $M$, Setälä K, Kivelä T, Haltia $M$, Norio $R$. The PEHO syndrome (progressive encephalopathy with PEHO syndrome (progressive encephalopathy with
edema, hypsarrhythmia, and optic atrophy): ophthalmoedema, hypsarrhythmia, and optic atrophy): ophthalmo-
logical findings and differential diagnosis. Neuro-ophthallogical findings and differ

5 Stibler H, Jaeken J. Carbohydrate deficient serum transferrin in a new systemic hereditary syndrome. Arch Dis Child 1990;65:107-11.

6 Li CC. Human genetics: principles and methods. New York McGraw-Hill, 1961:58-78.

7 Sorva R, Lankinen S, Tolpannen EM, Perheentupa J Variation of growth in height and weight of children. II Acta Paediatr Scand 1990;79:498-506.

8 O'Brien JS. $\beta$-galactosidase deficiency; ganglioside sialidase deficiency. In: Scriver CR, Beaudet AL, Sly WL, Valle $D$. The metabolic basis of inherited disease. Part II New York: McGraw-Hill, 1989:1797-806.

9 Graham JM. Smith's recognizable patterns of human deformation. 2nd ed. Philadelphia: Saunders, 1988:151, 157.

10 Norio R. Diseases of Finland and Scandinavia. In: Rothschild $\mathrm{H}$, ed. Biocultural aspects of disease. New York: child H, ed. Biocultural aspects

11 Norio R, Nevanlinna HR, Perheentupa J. Hereditary diseases in Finland; rare flora in rare soil. Ann Clin Res 1973;5:109-41. 